\title{
REVIEW
}

\section{New Exploration of Logic Starting Point of Audit Theory Structure}

\section{Zanchun Xie*}

Southwest Minzu University, Chengdu, Sichuan, 610041, China

\begin{tabular}{l}
\hline ARTICLE INFO \\
\hline Article history \\
Received: 6 September 2019 \\
Revised: 13 September 2019 \\
Accepted: 24 October 2019 \\
Published Online: 31 October 2019 \\
\hline Keywords: \\
Audit \\
Theoretical structure \\
Theoretical feature \\
Logical starting point
\end{tabular}

\section{Introduction}

The discipline of audit has existed since ancient times in China, but the development of modern audit has only been one hundred years. The theory of audit is very perfect. Although it has a relatively complete and huge theoretical system, the logical starting point selection element of its theoretical structure is still not reached consensus within the research scholars. There are many opinions on how to select the starting point of the audit theory structure in the research of audit theory at home and abroad. This paper analyzes and explores this issue, organizes various perspectives on it, and summarizes the author's own views, providing reference for relevant researchers.

\section{The Characteristics Should Be Met by the Logical Starting Point of the Audit Theory Structure}

The so-called logical starting point in the philosophi-

\begin{abstract}
As a discipline that has developed for many years, audit has been relatively mature and complete in its theoretical structure. As we all know, the establishment of any discipline requires a set of mature theoretical framework that are logical, progressive from point to surface. This theoretical structural framework is called the theoretical structure, and the research process of the audit discipline also needs to build such a theoretical system to make the audit research planned and structured, then the logical starting point in the audit theory structure will largely influence the direction and development of the audit discipline. In this paper, by expounding the characteristics and conditions of the logical starting point and combining some domestic and international discussions on the logical starting point, the author considers and explores the selection of logical starting point of the audit theory structure, hoping to select an amenable element as the logical starting point of the theoretical system to promote the research and development of the audit discipline.
\end{abstract}

cal explanation is the starting point or rising point that something rises in the process from the abstract meaning to the concrete meaning, and what we usually research is its selection and scope. In the research process of any discipline, it is necessary to specifically select and determine the logical starting point of its theoretical structure to ensure the subsequent development of this discipline and the construction of the theoretical system. The logical starting point selection of the audit theory structure needs to be more cautious. The selection of the logical starting point will have a great impact on the practical application of the audit work. Therefore, more accurate and scientific theoretical guidance is needed, based on past researches, the logical starting point of the audit theory structure need to meet the following characteristics.

\subsection{Easy to Understand}

The logical starting point of the audit theory structure

*Corresponding Author:

Zanchun Xie,

Southwest Minzu University, No. 16 South Fourth Section, First Ring Road, Wuhou District, Chengdu, Sichuan, 610041, China; E-mail: 188294716@qq.com. 
should be the most basic and fundamental part of the entire audit theory system structure, which is basic, highly general and easy to understand. As the research starting point of the audit discipline, if it is impossible for people to clearly read its meaning, subsequent audit research and study cannot be carried out. Therefore, first the logical starting point of the audit theoretical structure must be simple and basic, laying a good foundation for the subsequent audit theory structure.

\subsection{Connectivity}

Since it is the starting point of the theoretical structure, it must be able to connect and extend the theoretical structure, rather than independent existence, so the logical starting point of the audit theory structure needs to use this as a starting point to extend other structural elements. This is an important condition to be met to establish a complete theoretical structure. It not only needs to link the audit work practice with the theoretical structure, but also bears the role of linking the elements of other audit frameworks in the audit system. From this starting point, we can infer other structural elements, and each element can be logically and systematically developed, and the structure of the audit theory revealed in this way will be complete, organized and systematic.

\subsection{Practicableness}

No discipline can exist independently from practice, and audit is no exception. In the process of researching the logical structure of audit theory, it is inevitably to summarize and research combining with the experience in practice. As a logical starting point of the structure, it needs to be able to connect the audit theory and practice, so as to reveal the contradiction between its various elements and use audit examples to verify it, which makes the research of the audit discipline cannot be out of touch with the practice and the study of the theory is more meaningful.

\subsection{Development}

The discipline of audit has existed since ancient times. It is also a continuous developed discipline at present and in the future. Then the logical starting point of the audit theory structure needs some forward-lovingness and development, which can assist its future research and development. It can make corresponding adjustments in accordance with the changes of development trends and situations to provide proper theoretical basis for practical application and to continuously promote and guide the development of audit practice work.

\section{The Viewpoint Established by the Logical Starting Point of the Audit Theory Structure}

At present, there are mainly four viewpoints on the logical starting point of the audit theory structure at home and abroad. They take the audit hypothesis, the audit goal, the audit nature and the audit environment as the logical starting point of the audit theory structure. The following four perspectives will be analyzed separately.

\subsection{Taking the Audit Hypothesis as the Logical Starting Point of the Audit Theory Structure}

The audit hypothesis starting point theory was put forward by the United States auditor Shandell. In his own works, he discussed the viewpoint of audit hypothesis starting point theory. This viewpoint holds that the audit hypothesis is a basic proposition and it represents the in-depth definition of the audit theory structure, and also it is the basis of the audit practice process.

As an objective environment, it is binding on the research of the audit discipline. Therefore, the theoretical structure of the audit discipline should be based on the audit hypothesis, that is, through the audit hypothesis most people can identify, to determine the principles of the audit, so as to derive the entire audit theory structure and general process to determine the audit principle after the audit hypothesis, and then describe the audit concept on this basis, and then elaborate the guidelines and specifications of audit. Used as the structure of the audit theory, audit hypothesis has certain advantages.

Through the hypothesis, the theoretical study of the audit can be established in the work practice, and the deductive method can be used to infer the theoretical structure of the audit. However, this inference has some subjectivity. The combination of theory with practice is often prone to problems, and also the audit hypothesis is inferred by the researchers based on the some facts that are not entirely convincing. Therefore, this kind of assumption and inference can be determined in a certain objective and stable environment. Based on this trait, the starting point theory of audit hypothesis is often used for the theoretical foundation of the audit theory structure rather than the logical starting point, and it is not accurate to use it as the logical starting point of the audit theory structure.

\subsection{Taking the Audit Goal as the Logical Starting Point of the Audit Theory Structure}

The viewpoint of audit goal the starting point theory is the described by Canadian auditor Anderson in his book. This view has been widely recognized in western countries that 
pursue pragmatic audit theory. The theoretical structural elements of the audit goal starting point theory are the audit goal, accredited audit standard, audit concept, public audit, audit technology and audit process. Western countries are more inclined to pay attention to the guiding significance of the audit theory system to the audit practice at that time. The audit goal starting point theory happens to be more valuable for practical application, and therefore it plays a key role in the audit work of Western countries. The audit goal can objectively show the requirements of the audit environment for the system.

As a system meeting the environmental standard, it can bridge the audit system and the environment to ensure that the audit system can achieve the audit goal. The communication bridge directs the operation of the auditing structural system to a large extent. The audit goal's sensitivity to the environment is relatively high, and it also has great significance to the practice. Therefore, it can also combine audit theory with practical application. However, this viewpoint also has certain flaws.

The audit goal starting point theory is usually more applicable to the guidance of audit practice, and can more clearly define the direction of audit work. But, in theory, as a logical starting point for the audit theory structure, it is difficult for the audit goal to achieve the required scientific rigor, and the theoretical level is low. It is difficult to unify the audit goals under different social circumstances, which will make the theoretical structure not comprehensive enough. Therefore, this theory can be applied to practical application and it is not applicable to be used as a logical starting point in theory.

\subsection{Taking the Audit Nature as the Logical Start- ing Point of the Audit Theory Structure}

The audit nature starting point theory has always been in an important position in the process of audit theory research in China. What this viewpoint needs to express is that the audit theory structure must start from its origin and can be used as a starting point to extend other conclusions. By fully understanding the nature and connotation of the audit discipline, we can logically infer the object, function, principle, and criterion of the audit, and use these as the theoretical structure of the audit discipline.

This view has been widely respected in China5s audit research. The nature of things determines the fundamental attributes of things, which also means that the nature of things is abstract. As a logical starting point of a theory, it needs the ability to connect systems and environment, practice and theory. And it cannot define a thing singly, which will have minimal significance in its practical work. The logical starting point we need to look for is to provide effective guidance for the practical work of audit, and we should use this as a starting point to infer the assumption and goal of audit. The abstract concept of nature makes it difficult for people to thoroughly understand auditing theory, which makes view out of touch with practice and cannot be better matched with environmental changes.

\subsection{Taking the Audit Environment as the Logical Starting Point of the Audit Theory Structure}

The audit environment starting point theory is to combine the internal and external environment of audit, so that the audit theory determines its goal and audit report from the external environment and influences the audit's working procedure and working method. From the point of internal environment, the audit's internal environment can decide the nature and function of the audit, so as to determine the audit's working procedure and method. The internal and external environments are common, and the audit goal, nature, and function can also be organically combined in the audit practice.

The audit environment has important influence on all aspects of the audit, but if we singly take the audit environment as the logical starting point, which is separated from other audit factors, it is easy to cause the audit environment to be disconnected from practice and out of touch with the nature. Then since the theory of audit should be derived from audit practice, and this theory needs to guide the development direction of audit and serve the society, so it is necessary to put the logical starting point of the audit theoretical structure into practice, and to integrate it with the external environment to carry out expansion and extension, so as to make the theoretical research of this discipline more meaningful. And the audit environment can influence the development direction of the audit to a great extent, but it also needs to be combined with more audit elements in many aspects.

\section{The Author's Opinion on the Logical Start- ing Point of the Audit Theory Structure}

In summary, we can easily find that any viewpoint has its advantages and disadvantages. As the logical starting point of the audit theory structure, every single element cannot make the theoretical structure of the audit to be extended and developed furthest. Then we can get the conclusion that the logical starting point of the audit theory structure cannot be unilateral, it can only circumvent the shortcomings by including multiple elements.

If we want the theoretical structure of the audit can be better expressed, the audit environment and goal need to be combined, in the situation of not departing from the 
nature of the audit, comprehensive factors will be taken as the logical starting point for the audit theory structure. The environment and goal of audit are the main elements. This is largely due to the fact that the audit environment and audit goal are known, rather than the elements that need to be forecasted. The audit environment can greatly affect the audit goal, and the combination of the two can estimate the assumption of the audit within a stable period of time. Therefore, this can increase its scientific and accuracy, and it can make the various elements of the audit be presented after deduction, and it is not difficult to reach the goal of extending the structure of the audit theory.

\section{Conclusion}

This paper analyzes the conditions that need to be satisfied in the logical starting point of the audit theory structure, and sorts out four domestic and international perspectives on the selection of starting points. After research and discussion, we believe that any element that exists alone does not conform to the requirements of logical starting point of the audit theory structure. If you want to make the logical starting point meet the requirements of discipline research and development to the maximum extent, you need to combine the audit environment and goal to determine the logical starting point of the audit theory structure, so as to meet the requirements of practice and discipline development to the logical starting point of the audit theory structure.

\section{References}

[1] Zhang Jing, Yang Zhiqiang. Rethinking on the Logical Starting Point of Auditing Theory Structure[J]. Research of Finance and Accounting, 2014(1):53-55.

[2] Li Xuesong. The Constructionof the Auditing Theory Structure with the New Auditing Function Viewpoint as the Logical Starting[J]. Commercial Accounting, 2016(18):55-57.

[3] Hou Xinxia. My Opinion on the Logical Starting Point of Auditing Theory Structure[J]. Finance and Accounting Monthly, 2013(3a):27-28.

[4] Duan Ye. Research on Logical Starting Point of Auditing Theory Structure Based on Dialectical Materialism[J]. Manager Journal, 2016(10):154-155.

[5] Gao Jie, Gao Linyu. Exploration on Problems of Research Starting Point and Logical Starting Point of Management Studies[J]. Journal of Dongbei University of Finance and Economics, 2011(3):11-14.

[6] Yao Fushan. Research on the Internal Auditing Theory Structure Based on Motivation Origin[J]. Internal Auditing in China, 2016(12):8-12.

[7] MT Henderson. Corporate Heroin: A Defense of Perks, Executive Loans, and Conspicuous Consumption.Social Science Electronic Publishing[J]. Internal Auditing in China, 2018(10):4.

[8] C Mand.RE Duncan.L Gillam,Genetic selection for deafness: the views of hearing children of deaf adults.Journal of Medical Ethics[J]. 2018(11):18-25. 